\title{
A mechanism to account for well known peculiarities of low mass AGB star nucleosynthesis
}

\author{
Sara Palmerini ${ }^{1,2, \star}$, Oscar Trippella ${ }^{1,2}$, Diego Vescovi ${ }^{1,2}$, and Maurizio Busso ${ }^{1,2}$ \\ ${ }^{1}$ Dipartimento di Fisica e Geologia, Università degli Studi di Perugia, Italia \\ ${ }^{2}$ INFN, Sezione di Perugia, Italia
}

\begin{abstract}
We present here the application of a model for a mass circulation mechanism induced by the stellar magnetic field to study peculiar aspects of AGB star nucleosynthesis. The mixing scheme is based on a previously suggested magnetic-buoyancy process $[1,2]$ and here shown to account adequately for the formation of the ${ }^{13} \mathrm{C}$ neutron source for s-processes. In particular our analysis results are focused on addressing the constrains to AGB nucleosynthesis coming from the isotopic composition of presolar grains recovered in meteorites. It turns out that $n$-captures driven by the magnetically-induced mixing can account for the isotopic abundance ratios of $s$-elements recorded.
\end{abstract}

\section{Introduction}

The Asymptotic Giant Branch (AGB) phase is a late stage of evolution of low mass stars $\left(\mathrm{M} \leq 6 \mathrm{M}_{\odot}\right)$ that is well known to be site for the production of nuclei heavier than Fe via the slow neutron capture process ( $s$-process). Two reactions, the ${ }^{13} \mathrm{C}(\alpha, \mathrm{n}){ }^{16} \mathrm{O}$ and the ${ }^{22} \mathrm{Ne}(\alpha, \mathrm{n})^{25} \mathrm{Mg}$, are the sources of neutrons for nucleosynthesis of AGB stars. Since the ${ }^{22} \mathrm{Ne}(\alpha, \mathrm{n})^{25} \mathrm{Mg}$ can not be efficiently burnt in stars with mass smaller than $3 \mathrm{M}_{\odot}$ because of the low temperatures, the $s$-elements from $\mathrm{Sr}$ to $\mathrm{Pb}$ observed in low mass AGB stars have then to be produced trough the capture of neutrons provided by the ${ }^{13} \mathrm{C}$ source. The crucial point is to understand how a congruous amount of ${ }^{13} \mathrm{C}$ can form in the He-rich region of these stars. The mechanism suggest years ago by [3] and widely accepted is that protons are injected from the envelope into the He-rich layers at the moment of the third dredge-up (hereafter TDU), namely when at the end of a thermal instability due to a He-burning episode the convection penetrates into the radiative stellar region and brings to the stellar surface fresh synthesized materials. Afterwards, when the $\mathrm{H}$-shell burning restarts, protons penetrated below the edge of the convection yield to the formation of ${ }^{13} \mathrm{C}$ in the He-rich region via the ${ }^{12} \mathrm{C}(\mathrm{p}, \gamma){ }^{13} \mathrm{~N}\left(\beta^{+}\right){ }^{13} \mathrm{C}$ reaction chain, later followed by the activation of the ${ }^{13} \mathrm{C}(\alpha, n){ }^{16} \mathrm{O}$ reaction. Notice that the relation between the quantity of mixed protons and the number of nuclei synthesized by $s$-processing is not linear, indeed if too much protons are injected in the zone of interest ${ }^{14} \mathrm{~N}$ forms rather than ${ }^{13} \mathrm{C}$ and it will hamper the production of heavy nuclei because of its particularly large neutron capture cross section.

Until recently, despite decades of studies and the observational evidence that $s$-process elements are produced by low mass stars ascending the second giant branch, the formation of the so called

\footnotetext{
^e-mail: sara.palmerini@pg.infn.it
} 
${ }^{13} \mathrm{C}$ - pocket has not been explained by self-consistent models, forcing researchers to extensive parameterizations. In the last years mass circulation mechanism induced by stellar magnetic field have been studies by several authors (eg. [4, 5]). In particular [1] demonstrated that in red giant stars magnetized parcels of matter might arrange themselves in buoyant structures that expand from radiative layers to convective envelopes. [2] shown this process to be a reliable trigger for the proton injection in the He-rich region during the TDU, then for the ${ }^{13} \mathrm{C}$ - pocket formation. Even if in this scenario the number of mixed protons is smaller than what usually claimed in literature [6], the model well reproduces the s-element abundances measured in young open clusters and on C-rich AGB stars. This is so because the s/C ratio in the material dredged-up from the He-shell to the envelope is significantly larger than in parametric models (see e.g., [7] and [8]) thanks to s different slope of the proton injection, which allows the formation of a ${ }^{13} \mathrm{C}$ reservoir of several $10^{-3} \mathrm{M}_{\odot}$ but a smaller production of ${ }^{14} \mathrm{~N}$.

In this paper we compare results of our model for n-captures driven by magnetically-induced mixing with records of isotopic abundance ratios of s-elements in presolar $\mathrm{SiC}$ grains, which offer precise constraints on AGB nucleosynthesis and on the ${ }^{13} \mathrm{C}$ - pocket in particular.

\section{Nucleosynthesis models, grains and results}

Following the approach outlined by [2,9] and [10], we computed the proton penetration into the He-rich layers, the consequent formation of the ${ }^{13} \mathrm{C}$ reservoir and the s-element nucleosynthesis for a set of selected AGB stellar models with mass from 1.5 to $3 \mathrm{M}_{\odot}$ and metallicity from $1 / 3$ to 1 solar. With this small database of stellar nucleosynthesis, we tried to reproduce the relative distributions of s-process isotopes and elements, as measured in $\mathrm{SiC}$ grains. We used for comparisons the sample of grains analyzed by [11] and the data about Mainstream SiC grains present in the on-line Presolar Grain Database of the Washington University in St. Louis ([12] and subsequent updates).

The comparisons between our model predictions for isotopic and elemental ratios, pulse after pulse, and the measured data are shown in Figure 1. The model lines are characterized by dots that represent the various TPs. We show results for the isotopic ratios of $\mathrm{Zr}$ and $\mathrm{Ba}$ as the most rappresentative ones among light s- $(l s)$ and heavy s- $(h s)$ elements, respectively. Despite the large error bars of grain data, Figure 1 presents a satisfactory agreement between models and measurements: model curves fit the $\mathrm{SiC}$ isotopic abundances with the same accuracy of the best results achieved by other authors and available in the literature [11, 13-15]. In general, we account for the measurements of $\mathrm{Ba}$ isotopes at the same level of accuracy of the quoted authors $([13,14])$. Perhaps the discrepancy between data and calculations in the lowermost part of the measured points in panel $\mathrm{C}$, which is not well fitted (neither by us, nor by previous works) might hints to a problem in the nuclear parameters controlling the nucleosynthesis of s-process isotopes. Moreover, the presented AGB models reach the low values of ${ }^{91} \mathrm{Zr} /{ }^{94} \mathrm{Zr}$ and the high ones of ${ }^{96} \mathrm{Zr} /{ }^{94} \mathrm{Zr}$ and ${ }^{92} \mathrm{Zr} /{ }^{94} \mathrm{Zr}$ isotopic ratios better than before, although not all the points are reproduced. This is so because neutron captures at low neutron density are favored by the extension and the shape of the "magnetically" formed ${ }^{13} \mathrm{C}-$ pocket, which permit also a better reproduction of $\mathrm{Sr}$ isotopic ratios, as already illustrated in panel $\mathrm{F}$ and in [2]. Since a crucial constraint on the ${ }^{13} \mathrm{C}$ - pocket (its distribution and form) are the relative trends of $l s$ and $h s$ nuclei [11], we plot $\mathrm{Zr}$ versus $\mathrm{Ba}$ isotopic ratios in panel $\mathrm{D}$ and $\mathrm{E}$, where our model curves account for almost the whole area covered by the data points. This good agreement for $\mathrm{Zr}$ and $\mathrm{Ba}$ is the most important result in this work, because it proves that magnetically-induced mixing can yield for the formation of a ${ }^{13} \mathrm{C}$ - pocket, whose characteristics are fixed by the stellar physics and cannot be further fine tuned, that fulfill the AGB nucleosynthesis constraints of $\mathrm{SiC}$ grain composition. This result comes with an extra property: the same model of magnetic induce mixing when applied to the radiative layers above the $\mathrm{H}$-burning shell reproduces also the isotopic abundances of nuclei affected 

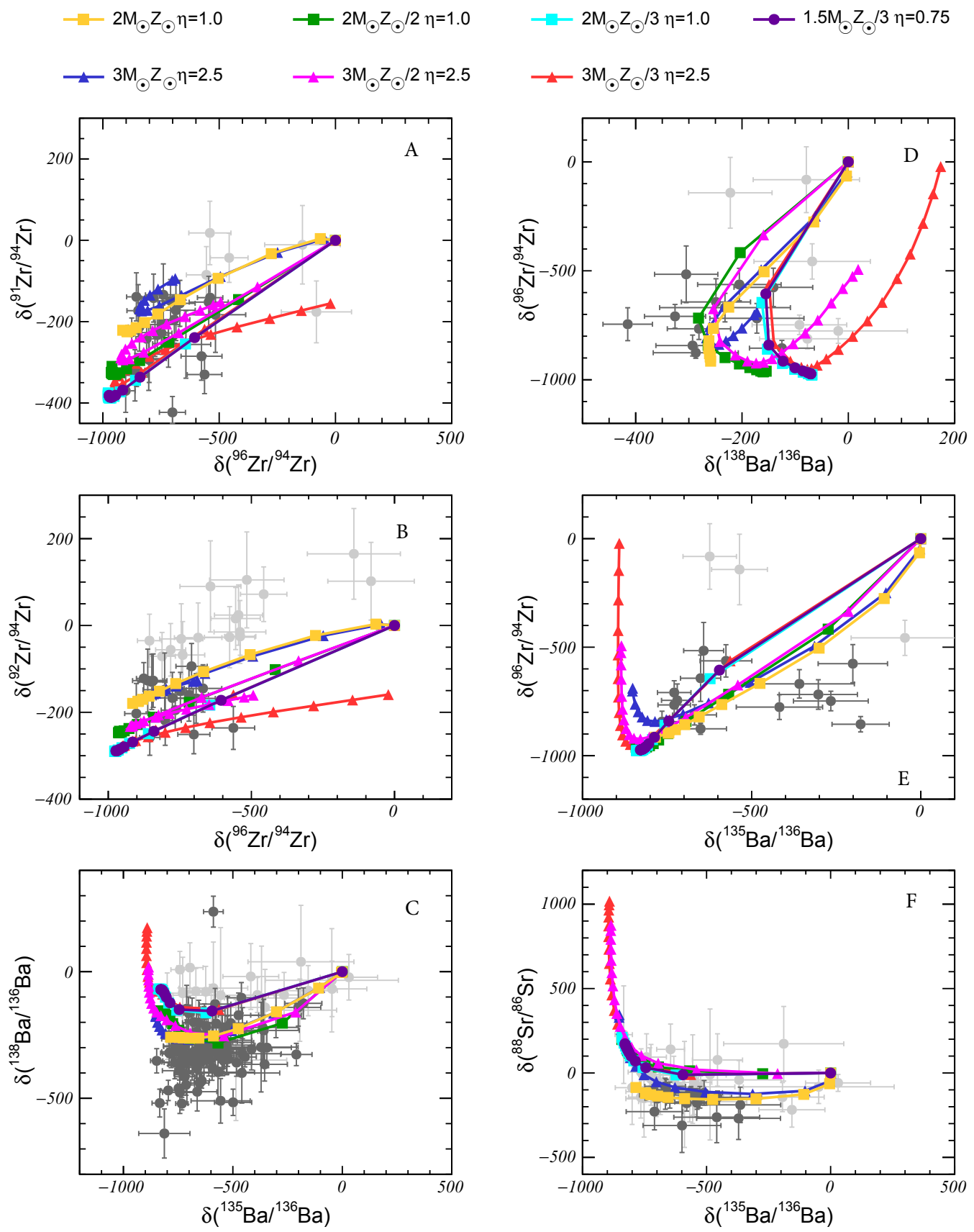

Figure 1. Comparison between our model predictions and presolar-grain relative isotopic abundances. The plot reports $\delta$ (per mill) values for $\mathrm{Zr}$, Ba and $\mathrm{Sr}$ isotopic ratios. Data points (full dots) refer to measurements from [11] and [12]. We report in a darker color the grains having error bars smaller than the $70 \%$ of the measurements themselves. The curves show the evolution in time of the abundances in the stellar envelopes; the dots along the lines represent the various TPs. Model calculations are for AGB stars with mass from 1.5 to $3 \mathrm{M}_{\odot}$ and metallicity from $1 / 3$ to 1 solar. 
by proton captures, typical of evolved low mass stars [9], therefore ours sees to be the unique approach for the moment to offer a rather general tool for treating deep mixing in evolved red giants.

\section{References}

[1] Nucci, M. C. \& Busso, M., ApJ 787, 141 (2014)

[2] Trippella, O., Busso, M., Palmerini, S. et al., ApJ 818, 125 (2016)

[3] Straniero, O., Gallino, R., Busso, M., et al., ApJ 440, L85 (1995)

[4] Denissenkov, P. A., \& Merryfield, W. J., ApJ 727, L8 (2011)

[5] Busso, M., Wasserburg, G. J., Nollett, K. M., \& Calandra, A., ApJ 671, 802 (2007)

[6] Maiorca, E., Magrini, L., Busso, M., et al., ApJ 747, 53 (2012)

[7] Busso, M., Gallino, R., \& Wasserburg, G. J., ARA\&A 37, 239 (1999)

[8] Bisterzo, S., Travaglio, C., Gallino, R., et al., ApJ 787, 10 (2014)

[9] Palmerini,S., Trippella, O. \& Busso, M., MNRAS 467, 1193 (2017)

[10] Palmerini,S., Trippella, O. , Busso, M., et sl. GeoCoA in press (2017)

[11] Liu N., Savina M. R. \& Gallino R., et al. , ApJ 803, 12 (2015)

[12] Hynes, K.M. \& Gyngard, F., LPSC XL, Abstract 1198, (2009)

[13] Lugaro M., Tagliente G., Karakas A. I., et al., ApJ 780, 95 (2014)

[14] Liu, N., Gallino, R., Bisterzo, S., et al. ApJ 786, 66 (2014).

[15] Liu, N., Gallino, R., Bisterzo, S., et al. ApJ 788, 66 (2014). 